\title{
SIMULATION OF CHARGED PARTICLE TRANSPORT IN NONLINEAR AXISYMMETRICAL ELECTROSTATIC POTENTIAL
}

\author{
I.P. Yudin, V.V. Andreev \\ Joint Institute for Nuclear Research, Dubna 141980, Moscow region, Russia
}

Abstract

We describe the procedure within transfer matrix formalism to obtain the solution of nonlinear charge particle motion equation in the electrostatic fields (with axis-symmetrical potential) up to third-order aberrations.

\section{INTRODUCTION}

In this paper we describe the procelure within transfer matrix formalism to obtain the solution of nonlinear charge particle motion equation up to third-order aberrations in the electrostatic fields.

The matrix elements are given with the coefficients in the Taylor expansion,

$$
\begin{aligned}
& X_{i}(z)=\sum_{j=1}^{4} R_{i j}(z) X_{j}^{0}+\sum_{j=1}^{4} \sum_{k=j}^{4} T_{i j k}(z) X_{j}^{0} X_{k}^{0}+ \\
& +\sum_{j=1}^{4} \sum_{k=j}^{4} \sum_{l=k}^{4} U_{i j k l}(z) X_{j}^{0} X_{k}^{0} X_{l}^{0}+\ldots
\end{aligned}
$$

where $\vec{X}$ is the usual 4-vector of the TRANSPORT [1] coordinates, $\vec{X}=\left(r, r^{\prime}, l, \delta\right)$. The same description of our method can be found in [2] for magnetic solenoid lenses. This paper brings up the investigation that it concludes in $[3,4]$.

\section{NONLINEAR MOTION EQUATION}

In cylindrical coordinate system with the $\mathrm{Z}$ - axis directed straight along the $\vec{E}$ axial field, the nonlinear motion equation originates from the Lorentz force equation for the charged particles in the external axis-symmetric field [5].

$$
\left\{\begin{array}{l}
d / d t(\gamma m \dot{r})-\gamma m r \dot{\theta}^{2}-q E_{r}=0, \\
d / d t(m \dot{z})-q E_{z}=0, \\
d / d t\left(m r^{2} \dot{\theta}\right)=0,
\end{array}\right.
$$

where $m$ is the particle rest mass, $q$ is the particle charge, $\gamma=1 / \sqrt{1-\beta^{2}}, \beta=v / c, v-$ the particle velocity, $\mathrm{c}-$ the speed of light in vacuum, $E_{r} \& E_{z}$ are the radial and axial components of the electrostatic field:

$$
\left\{\begin{array}{l}
E_{z}(r, z)=-\Phi^{\prime}(z)+r^{2} / 4 \Phi^{\prime \prime \prime}(z)-\ldots, \\
E_{r}(r, z)=r / 2 \Phi^{\prime \prime}(z)-r^{3} / 16^{\prime \prime \prime \prime}(z)+\ldots .
\end{array}\right.
$$

Eqs. (1.2a) - (1.2b) were written from known relation $\vec{E}=-\nabla \varphi$ and the decomposition $\varphi(r, z)$ on degree $r$ and $z$ with the axis-symmetrical condition $\varphi(r, z)=-\varphi(-r, z)$ :

$$
\varphi(r, z)=\Phi(z)-\frac{r^{2}}{4} \Phi^{\prime \prime}(z)+\frac{r^{4}}{64} \Phi^{\prime \prime \prime \prime}(z)-\ldots,
$$

$\Phi(z)=\varphi(0, z)$ is the axial electrostatic potential. The differentiation is held on $z$. For this investigation we describe $\Phi(\mathrm{z})$ as a piece-unterrupted (linear and square) function. To obtain the nonlinear motion equation, we have written the conservation law of the energy from behind a nonconservation of our investigation system:

$$
V^{2}=\dot{r}^{2}+\dot{z}^{2}=\frac{2|q|}{\gamma m} \varphi(r, z)
$$

We suppose

$$
\left\{\begin{array}{l}
V_{z}>V_{r}, \text { and } \\
r^{\prime}=\frac{\dot{r}}{\dot{z}}<1
\end{array}\right.
$$

then Eq. (1.4) can be written in the following form

$$
\dot{z}^{2}=\frac{2|q|}{\gamma m} \varphi(r, z)\left[1-r^{\prime 2}\right],
$$

taking into account that the decomposition up to the third order. Thus, by the elimination of a time dependence in (1.1b), and then by the substitution of Eq. (1.6) with the decomposition of Eq. (1.3) truncated after $r^{3}$, the main nonlinear equation of the particle trajectory in the electrostatic field with axial potential $\Phi(z)$ (on closed interval $[0, D]$ ) can be written in the form:

$$
\begin{gathered}
2 \operatorname{sign}(q) \cdot \Phi r^{\prime \prime}-\Phi^{\prime} r^{\prime}-\frac{\Phi^{\prime \prime}}{2} r=\left(\frac{\Phi^{\prime \prime 2}}{8 \Phi}-\frac{\Phi^{\prime \prime \prime \prime}}{16}\right) r^{3}+ \\
+\left(\frac{\Phi^{\prime} \Phi^{\prime \prime}}{4 \Phi}+\frac{\Phi^{\prime \prime \prime}}{4}\right) r^{2} r^{\prime}+\frac{\Phi^{\prime \prime}}{2} r r^{\prime 2}+\Phi^{\prime} r^{\prime 3} .
\end{gathered}
$$

In the case the magnitudes of $r$ and $r^{\prime}$ have remained small (near the axis), we obtain the paraxial particle linear equation:

$$
2 \operatorname{sign}(q) \cdot \Phi r^{\prime \prime}-\Phi^{\prime} r^{\prime}-\Phi^{\prime \prime} / 2 \cdot r=0 \text {. }
$$

In further we are limited the $\operatorname{sign}(q)=-1$ in Eq. (1.7), i.e., we describe the motion of an electron far from the axis. Into the decreasing electrostatic field the trajectory of a proton will be the same as one for an electron in the increasing (in the same of a degree) field. It follows from Eq. (1.7), thus one is not dependent explicitly on the $q / m$ ratio.

The basis of our solution method of Eq. (1.7) is the expression (1). Independent variables are $\vec{X}=\left(r, r^{\prime}, l, \delta\right)$. Here is the $l$-definition:

$$
l=\int_{0}^{z}\left(\sqrt{1+r^{\prime 2}+r^{2} \theta^{\prime 2}}-1\right) d z \approx 1 / 2 \int_{0}^{z} r^{\prime 2} d z .
$$

The appearance of a transverse velocity is to influence on the beam dispersion increment: 


$$
p=p_{0} \cdot(1+\delta)=m v_{z} \cdot\left(1+1 / 2\left(v_{r} / v_{z}\right)^{2}\right)=p_{0} \cdot\left(1+r^{\prime} 2 / 2\right)
$$

After substituting Eq. (1.2) into Eqs. (1.1a) and (1.1b) and then the result - into Eq. (1.10) we can write in third order:

$$
\delta=\frac{\Phi^{\prime \prime}}{8 \Phi} \cdot r^{2}
$$

The aberration coefficients $R_{i j}, T_{i j k}$ and $U_{i j k l}$ define the first, the second and the third orders of transfer matrix and of system properties at all. We shall use some useful relations for "primes" and "nonprimes":

$$
R_{2 j}(z)=R_{1 j}^{\prime}(z), T_{2 j k}(z)=T_{1 j k}^{\prime}(z), U_{2 j k l}(z)=U_{1 j k l}^{\prime}(z) .
$$

To define $f_{i j k}^{T}, f_{i j k l}^{U}$ as the appropriate right parts of the main trajectory Eq. (1.7), it can written the beginning condition for the definition of aberration coefficients:

$$
\left\{\begin{array}{l}
T_{1 j k}(0)=T_{1 j k}^{\prime}(0)=0, T_{2 j k}(0)=T_{1 j k}^{\prime}(0)=0, \\
T_{2 j k}^{\prime}(0)=T_{1 j k}^{\prime \prime}(0)=f_{1 j k}^{T}(0) . \\
U_{1 j k l}(0)=U_{1 j k l}^{\prime}(0)=U_{2 j k l}(0)=0, \\
U_{2 j k l}^{\prime}(0)=U_{1 j k l}^{\prime \prime}(0)=f_{1 j k l}^{U}(0) .
\end{array}\right.
$$

\section{LINEAR POTENTIAL}

Let observe the potential of the view:

$$
\Phi(z)=\Phi_{0} \cdot\left(1+z / z_{0}\right)=\Phi_{0} \cdot \bar{z},
$$

where $z_{0}=D /\left(n^{2}-1\right), n=\sqrt{\Phi_{1} / \Phi_{0}}, D$ is the length of the acting field region, $\Phi_{0}$ and $\Phi_{1}$ are initial and final potential values, $n$-electron-optics [5] index of the reflection that appropriate by the Snellius law. Naturally it impose a constraint on $n$ that electrostatic potential is equal to zero where the particle velocity is equal to zero.

\subsection{Third-order optics in an accelerating (decelerating) tube with a linear potential}

The first-order optics is described in [4,6] .

The main Eq. (1.7) with a linear potential (2.1) is adopted here as:

$$
2 \Phi r^{\prime \prime}+\Phi^{\prime} r^{\prime}=-\Phi^{\prime} r^{\prime 3}
$$

From that follows explicitly the contribution into $r$ and $r^{\prime}$ it gives only in the third order, i.e., $T_{1 j k}(z)=T_{2 j k}(z)=0$. By the substitution of linear soluton [4, 6] into Eqs. (1.9) - (1.10), we obtain the following non-zero components of the matrix $\mathrm{T}$ (with $\zeta=\sqrt{1+z / z_{0}}$ ):

$$
\left\{T_{322}(z)=z_{0} / 2 \cdot 1 n \zeta^{2}, T_{422}(z)=1 /\left(2 \zeta^{2}\right)\right. \text {. }
$$

We find the accurate analytical solution of Eq. (1.7) with the potential (2.1). In this case the initial equation of the third order can be written to substitute the linear potential (2.1) once into Eqs. (1.2)-(1.3) and then into Eq. (1.1):

$$
2 \bar{z} r^{\prime \prime}+\left(1+r^{\prime 2} / z_{0}^{2}\right)=0
$$

where we are accounting the obvious condition (1.5). The directly integration Eq. (2.1.3) on $z$ with the initial conditions $\left(r_{0}, r_{0}^{\prime}\right)$ gives us the following nonlinear solution:

$$
\left\{\begin{array}{l}
r^{\prime}(z)=\frac{r_{0}^{\prime}}{\sqrt{\zeta^{2}\left(1+r_{0}^{\prime 2}\right)-r_{0}^{\prime 2}}} \\
r(z)=r_{0}+\frac{2 z_{0} r_{0}^{\prime}}{1+{r_{0}^{\prime}}^{2}} \cdot\left[\sqrt{\zeta^{2}\left(1+r_{0}^{\prime 2}\right)-r_{0}^{\prime 2}}-1\right] .
\end{array}\right.
$$

By using Eq. (1.5), to reduce this result solution to view (1) we have the accurate solution of Eq. (2.1.3):

$$
\left\{\begin{array}{l}
r^{\prime}(z)=\frac{r_{0}^{\prime}}{\zeta} \cdot\left(1-\frac{r_{0}^{\prime 2}}{2} \cdot \frac{z / z_{0}}{1+z / z_{0}}\right), \\
r(z)=r_{0}+2 z_{0} r_{0}^{\prime}[\zeta-1]\left(1-r_{0}^{\prime 2} / 2 \cdot(\zeta-1) / \zeta\right) .
\end{array}\right.
$$

is written as

$$
\left(\begin{array}{c}
r \\
r^{\prime}
\end{array}\right)=\left[\mathbf{R}+\mathbf{U}_{\mathbf{2 2}} \cdot r_{0}^{\prime 2}\right] \cdot\left(\begin{array}{c}
r_{0} \\
r_{0}^{\prime}
\end{array}\right),
$$

where $\mathbf{R}=\left(\begin{array}{cc}R_{11} & R_{12} \\ 0 & R_{22}\end{array}\right), \mathbf{U}_{\mathbf{2 2}}=\left(\begin{array}{ll}0 & U_{1222} \\ 0 & U_{2222}\end{array}\right)$.

Fringe region focusing effects change also in the third order.

\section{SQUARE POTENTIAL}

Further we shall take into account the following potential:

$$
\Phi(z)=\Phi_{0}\left(1 \pm \bar{z}^{2}\right)
$$

where $\bar{z}=z / z_{0}, z_{0}=D / \sqrt{\left|n^{2}-1\right|}, n \neq 1$. We choose this potential so, that all derivatives of $\Phi(z)$ in the point $z=0$ were equal 0 . Sign $( \pm)$ in this case corresponds to electron acceleration $(+)$ or deceleration (-).

\subsection{The third-order beam optics of the acceleration tube with the square potential}

For first-order optics, the acceleration tube transfer matrix $R^{+}(z)$ and the deceleration tube transfer matrix $R^{-}(z)$ are described in [4].

In order to obtain nonzero elements of T-matrix we substitute the equation for $r^{\prime}(z)$ to the definition of the value $\delta$ from (1.13),

$$
\begin{aligned}
& z_{0}=D / \sqrt{n^{2}-1}(n>1), \zeta=1 / \sqrt{2} \cdot \text { Arsh } \bar{z}, \text { then: } \\
& T_{412}^{+}(\bar{z})=\frac{\sin ^{2} \zeta}{4 z_{0}^{2}\left(1+\bar{z}^{2}\right)}, \\
& T_{412}^{+}(\bar{z})=-\frac{\sin \zeta \cos \zeta}{2 z_{0}\left(1+\bar{z}^{2}\right)}, T_{422}^{+}(\bar{z})=\frac{\cos ^{2} \zeta}{4\left(1+\bar{z}^{2}\right)} .
\end{aligned}
$$

Integrating with the definition (1.9) gives the following nonzero elements of the third line of the T-matrix: 


$$
\left\{\begin{array}{l}
T_{311}^{+}(\bar{z})=\frac{1}{4 z_{0}^{2}} \cdot \int_{0}^{\bar{z}} \frac{\sin ^{2} t}{1+\tau^{2}} d \tau=\frac{1}{4 z_{0}^{2}} \cdot\left(\operatorname{arctg} \bar{z}-I_{1}\right), \\
T_{312}^{+}(\bar{z})=-\frac{1}{4 z_{0}} \cdot \int_{0}^{\bar{z} \sin t \cos t} \frac{I_{3}}{1+\tau^{2}} d \tau=-\frac{I_{3}}{4 z_{0}}, \\
T_{322}^{+}(\bar{z})=\frac{1}{4} \cdot \int_{0}^{z} \frac{\cos ^{2} t}{1+\tau^{2}} d \tau=\frac{I_{1}}{4},
\end{array}\right.
$$

where $t=1 / \sqrt{2} \cdot$ Arsh $\tau$. Eq. (1.7) with the square potential (3.1) can be written in the form:

$$
\begin{aligned}
& 2 \Phi r^{\prime \prime}+\Phi^{\prime} r^{\prime}+\frac{\Phi^{\prime \prime}}{2} r= \\
& -\frac{\Phi^{\prime \prime 2}}{8 \Phi} r^{3}-\frac{\Phi^{\prime} \Phi^{\prime \prime}}{4 \Phi} r^{2} r^{\prime}-\frac{\Phi^{\prime \prime}}{2} r r^{\prime 2}+\Phi^{\prime} r^{\prime 3}
\end{aligned}
$$

After substituting Eq. (3.1) into Eq. (3.1.3), combining same order members, we shall find driving forces to obtain third order aberration coefficients:

$$
\left\{\begin{array}{l}
f_{111}^{U}(\bar{z})=-\frac{\Phi_{0}}{2 z_{0}^{4}\left(1+\bar{z}^{2}\right)} \cdot\left(\cos \zeta-\frac{\sqrt{2} \cdot \bar{z}}{\sqrt{1+\bar{z}^{2}}} \cdot \sin \zeta\right), \\
f_{1112}^{U}(\bar{z})=-\frac{\Phi_{0} \cdot}{2 z_{0}^{3}\left(1+\bar{z}^{2}\right)} \cdot\left(\sin \zeta+\frac{\sqrt{2} \cdot \bar{z}}{\sqrt{1+\bar{z}^{2}}} \cdot \cos \zeta\right) .
\end{array}\right.
$$

Integrating with the Green's function gives us:

$$
\left\{\begin{array}{l}
U_{1111}^{+}(\bar{z})=z_{0}^{2} \cdot U_{1122}^{+}(\bar{z}), \\
U_{1112}^{+}(\bar{z})=z_{0}^{2} \cdot U_{1122}^{+}(\bar{z}) .
\end{array}\right.
$$

We introduce some useful formulas for beam parameters:

$$
\left(\begin{array}{c}
r \\
r^{\prime}
\end{array}\right)=\left[\mathbf{R}^{+}+\mathbf{U}_{\mathbf{1 1}}^{+} \cdot\left(r_{0}^{2}+r_{0}^{\prime 2} \cdot z_{0}^{2}\right)\right] \cdot\left(\begin{array}{c}
r_{0} \\
r_{0}^{\prime}
\end{array}\right),
$$

Where $\mathbf{R}^{+}=\left(\begin{array}{ll}R_{11}^{+} & R_{12}^{+} \\ R_{21}^{+} & R_{22}^{+}\end{array}\right), \quad \mathbf{U}_{11}^{+}=\left(\begin{array}{ll}U_{1111}^{+} & U_{1112}^{+} \\ U_{2111}^{+} & U_{2112}^{+}\end{array}\right)$.

Further, we shall integrate Eqs. (3.1.4) with the Green's function (4) according to relations (1.13). We obtained:

$$
\begin{aligned}
& U_{1111}^{+}(\bar{z})=-\frac{\Phi_{0}}{2 z_{0}^{2}} \cdot\left[\sin \zeta \operatorname{arctg} \bar{z}-I_{1} \sin \zeta+I_{3} \cos \zeta\right], \\
& U_{1112}^{+}(\bar{z})=-\frac{\Phi_{0}}{2 z_{0}} \cdot\left[\sqrt{2} \sin \zeta-I_{1} \cos \zeta-I_{3} \sin \zeta\right] \\
& U_{2111}^{+}(\bar{z})=-\frac{\Phi_{0}}{2 z_{0}^{3} \sqrt{2\left(1+\bar{z}^{2}\right)}} \cdot[\cos \zeta \operatorname{artcg} \bar{z}+ \\
& \left.+\sin \zeta \cdot \sqrt{2 /\left(1+\bar{z}^{2}\right)}-I_{2} \cos \zeta-I_{3} \sin \zeta\right], \\
& U_{2112}^{+}(\bar{z})=-\frac{\Phi_{0}}{2 z_{0}^{2} \sqrt{2\left(1+\bar{z}^{2}\right)}} \cdot\left[\sqrt{2} \cos \zeta \cdot\left(1-\frac{1}{\sqrt{1+\bar{z}^{2}}}\right)+\right. \\
& \left.+I_{1} \sin \zeta-I_{3} \cos \zeta\right] \text {, }
\end{aligned}
$$

3.2. The third-order beam optics of the deceleration tube with the square potential

After the same substitutions (see Section 3.1) we shall obtain following nonzero elements of the T-matrix:

$$
\left\{\begin{array}{l}
T_{411}^{-}(\bar{z})=\frac{s h^{2} \zeta}{4 z_{0}^{2}\left(1-\bar{z}^{2}\right)}, \\
T_{412}^{-}(\bar{z})=-\frac{s h \zeta c h \zeta}{2 z_{0}\left(1-\bar{z}^{2}\right)}, T_{422}^{-}(\bar{z})=\frac{c h^{2} \zeta}{4\left(1+\bar{z}^{2}\right)} .
\end{array}\right.
$$

$$
\left\{\begin{array}{l}
T_{311}^{-}(\bar{z})=\frac{1}{4 z_{0}^{2}} \cdot \int_{0}^{\bar{z}} \frac{s h^{2} t}{1-\tau^{2}} d \tau=\frac{1}{4 z_{0}^{2}} \cdot\left(I_{1}-\operatorname{Arth} \bar{z}\right), \\
T_{312}^{-}(\bar{z})=\frac{1}{4 z_{0}} \cdot \int_{0}^{\bar{z}} \frac{s h t c h t}{1-\tau^{2}} d \tau=\frac{I_{4}}{4 z_{0}}, \\
T_{322}^{-}(\bar{z})=\frac{1}{4} \cdot \int_{0}^{\bar{z}} \frac{c h^{2} t}{1-\tau^{2}} d \tau=\frac{I_{2}}{4},
\end{array}\right.
$$

where $z_{0}=D / \sqrt{1-n^{2}}(n<1), \quad \zeta=1 / \sqrt{2} \cdot \arcsin \bar{z}$, and $t=$ $1 / \sqrt{2} \cdot \arcsin \tau$.

We shall find driving forces to obtain third order aberration coefficients:

$$
\left\{\begin{array}{l}
f_{1111}^{U}(\bar{z})=-\frac{\Phi_{0}}{2 z_{0}^{4}\left(1-\bar{z}^{2}\right)} \cdot\left(\operatorname{ch} \zeta+\frac{\sqrt{2} \cdot \bar{z}}{\sqrt{1-\bar{z}^{2}}} \cdot \operatorname{sh} \zeta\right), \\
f_{1112}^{U}(\bar{z})=-\frac{\Phi_{0} \cdot}{2 z_{0}^{3}\left(1-\bar{z}^{2}\right)} \cdot\left(\operatorname{sh} \zeta+\frac{\sqrt{2} \cdot \bar{z}}{\sqrt{1-\bar{z}^{2}}} \cdot \operatorname{ch} \zeta\right) .
\end{array}\right.
$$

Integrating with the Green's function gives us:

$$
\left\{\begin{array}{l}
U_{1111}^{-}(\bar{z})=-z_{0}^{2} \cdot U_{1122}^{-}(\bar{z}), \\
U_{1112}^{-}(\bar{z})=-z_{0}^{2} \cdot U_{1222}^{-}(\bar{z}),
\end{array}\right.
$$

According to (1), we introduce some useful formula for beam parameters:

$$
\left(\begin{array}{c}
r \\
r^{\prime}
\end{array}\right)=\left[\mathbf{R}^{-}+\mathbf{U}_{\mathbf{1 1}}^{-} \cdot\left(r_{0}^{2}-r_{0}^{\prime 2} \cdot z_{0}^{2}\right)\right] \cdot\left(\begin{array}{c}
r_{0} \\
r_{0}^{\prime}
\end{array}\right),
$$

where $\mathbf{R}^{-}=\left(\begin{array}{ll}R_{11}^{-} & R_{12}^{-} \\ R_{21}^{-} & R_{22}^{-}\end{array}\right), \mathbf{U}_{11}^{-}=\left(\begin{array}{ll}U_{1111}^{-} & U_{1112}^{-} \\ U_{2111}^{-} & U_{2112}^{-}\end{array}\right)$.

Further, we shall integrate Eqs. (3.2.4) wiht the Green's function [4] according to relations (1.13). We found: 


$$
\begin{aligned}
& U_{1111}^{-}(\bar{z})=-\frac{\Phi_{0}}{2 z_{0}^{2}} \cdot\left[\operatorname{sh} \zeta \operatorname{Arth} \bar{z}-I_{2} \operatorname{sh} \zeta+I_{4} \operatorname{ch} \zeta\right], \\
& U_{1112}^{-}(\bar{z})=-\frac{\Phi_{0}}{2 z_{0}} \cdot\left[-\sqrt{2} \operatorname{sh} \zeta+I_{2} \operatorname{ch} \zeta-I_{4} \operatorname{sh} \zeta\right], \\
& U_{2111}^{-}(\bar{z})=-\frac{\Phi_{0}}{2 z_{0}^{3} \sqrt{2\left(1-\bar{z}^{2}\right)}} \cdot[\operatorname{ch} \zeta \operatorname{Arth} \bar{z}+ \\
& \left.+\operatorname{sh} \zeta \cdot \sqrt{2 /\left(1-\bar{z}^{2}\right)}-I_{2} \operatorname{ch} \zeta+I_{4} \operatorname{sh} \zeta\right], \\
& U_{2112}^{+}(\bar{z})=-\frac{\Phi_{0}}{2 z_{0}^{2} \sqrt{2\left(1-\bar{z}^{2}\right)}} \cdot\left[-\sqrt{2} \operatorname{ch} \zeta \cdot\left(1-\frac{1}{\sqrt{1-\bar{z}^{2}}}\right)+\right. \\
& \left.+I_{2} \operatorname{sh} \zeta-I_{4} \operatorname{ch} \zeta\right] \text {, }
\end{aligned}
$$

\section{CONCLUSION}

In this paper we describe the third-orer beam optics of the acceleration (decelration) tube, created with axis-symmetrical potential. We describe the linear and square electrostatic field models with nonlinear transfer matrices. The described procedure was taken into account to obtain numerical results of investigation of the $0.5 \mathrm{MeV}$ proton beam transport through the SPIN superconducting synchrotron injection channel [7]. Some modification of construction and channel parameters by choosing an optimal installation operation regime was proposed. A possibility of increasing the beam intensity at the entrance into the synchrotron ring was established by a computer simulation.

\section{REFERENCES}

[1] K.L. Brown et al. "TRANSPORT. A Computer Program for Designed Charged Particle Beam Transport Systems.” SLAC-91, Rev. 2, UC-28 (I/A), May 1977.

[2] V.V. Andreev, I.P. Yudin. "Third-order Beam Optics of Real Solenoid Lens" in: Proc. 5-th International Symposium on Electron Beam Ion Sources and Their Applications, (Dubna, USSR, Sept. 24-28, 1991), pp. 279-290. Editors: E.D. Donets, I.P. Yudin. JINR, Dubna, 1992.

[3] V.V. Andreev, I.P. Yudin. "The Full Matrices Construction of Linear Transformations of Accelerating Tube and Solenoid Magnetic Lens." JINR Communication B3-1-9231, Dubna, January 1992.

[4] V.V. Andreev, I.P. Yudin. "Linear Optics in Accelerating (Decelerating) Tube with a Piece-Unterrupted Potential," in: Proc. of Conference on Charged Particle Acclerators, (Dubna, Oct. 13-15, 1992), JINR D9-92-455, vol. II, pp. 81-88, Dubna, 1993.

[5] M. Sziladyi. "Electron and Ion Optics," New York, Plenum Press, 1988.

[6] J.R. Pierce. "Theory and Design of Electron Beams," Princeton: Van Nostrand, 1949, 1954.

[7] V.V. Andreev et al. "Proton Beam Loss Evaluation in the Injection channel of SPIN Synchrotron." JINR Communication P9-87-763, Dubna, October 1987. 